\title{
A general-purpose program for problem solving experimentation
}

\author{
ROBIN JEFFRIES and JOHN KARAT \\ University of Colorado, Boulder, Colorado 80309
}

\begin{abstract}
This paper describes a general program framework for carrying out experimentation in human problem solving. The framework is general enough to apply to other areas of research as well. A brief description of the major components of the program is given, and suggestions are offered for programmers in psychological research.
\end{abstract}

In writing programs to run real-time experiments, the choice is usually between using one of the experimentdescription languages and adapting one's needs to the language restrictions, or writing a program "from scratch" in FORTRAN, BASIC, or whatever language is available on the computer system. This paper describes a third possible approach: the design of a general specialpurpose program to handle a wide range of experiments within a given paradigm. We seek to demonstrate this approach by examining an experimental situation and pointing out the common features in experiments within that area. The experimental paradigm used here is human problem solving research. Although the following comments relate to problem solving, the program design will accommodate many types of experimentation.

The most common types of problems used in the study of human problem solving are those involving transformation. In these problems, a subject is presented a starting state, a goal state, and a set of rules for making moves to transform the problem from the start to the goal state. Problems of this type include water-jug problems, river-crossing problems, and Tower of Hanoi problems. Each of these requires the execution of a number of moves before the solution is reached. At any state in a problem, a decision may commonly be made between a number of possible legal and illegal moves.

In river-crossing problems, the subject is presented a task in which a number of characters are to be transported across a river in a boat. There are equal numbers of two types of travelers, and the boat has a limited capacity. A restriction on the selection of moves is that the "bad" characters are never allowed to outnumber the "good" characters on either side of the river. Any moves that result in such a situation are not permitted.

Water-jug problems present the solver with three containers of different sizes. The largest container is

This work was supported in part by NSF Grant BNS77-0679 and was performed using the facilities of the Computer Laboratory for Instruction in Psychological Research, which is supported in part by the University of Colorado. initially filled with water, and the task is to equally divide the water between the largest and the middlesize containers. The division takes place by pouring water from one container to another. Each of these operations continues until either the container being poured into is full, or the container that the water is being poured from is empty.

In Tower of Hanoi problems, the subject is presented a number of graduated disks positioned in a tower on one of three pegs. The disks are to be transferred to form a similar tower on another of the pegs by making a series of moves. Each move transfers a single disk from the top of the tower on one peg, to the top of the stack on another. At no time is the solver allowed to place a disk on top of a disk smaller than it is.

There are a number of ways in which these problems differ in structure, which makes each of them interesting to a cognitive psychologist. However, it is possible to describe the solutions to these problems in terms of their common features to demonstrate that this type of research does not require a program control structure much different from other areas. Quite simply, for each problem state presented to a subject, a response (move selection) is made. This response is recorded, and a decision is made by the program concerning the next problem state to be presented. Thus, the design of a problem solving program is actually very similar to the design of any program in which the flow of stimulus presentation is contingent upon some response.

If we were only interested in the presentation of a single problem and recording the solution to that problem, the design of a program would be fairly simple. However, we are attempting to study additional aspects of problem solving. As a first step, we are interested in modeling the performance of human subjects in novel problem solving tasks, and to study this behavior, we are interested in presenting a problem and recording the moves made by subjects in solving the task. In addition, we are interested in how a subject's solution path changes over multiple presentations of a problem, as well as how the solution of one problem affects the solution of a second problem presented after a number of trials on the first (learning or transfer effects). We 
have also examined notions about the development of complex plans in problem solving by examining the move latency patterns.

Our problem solving experiments follow the same general procedure. Using a CRT display terminal, a subject is presented a series of instructions, followed by the presentation of one or more "puzzles," each of which must be solved a predetermined number of times. This type of experiment is not suitable for programming in experiment-description languages such as SCAT (Stadler, 1969) or DTES (Spear, Overgard, \& Christian, 1975), since a given state in a transformation problem might be followed by a large number of other states. The experiment-description languages require that all paths be explicitly indicated, a process too cumbersome to be practical in this research. We are thus left with the task of writing our real-time experimental programs in FORTRAN IV.

The task of writing these programs has been made easier by the CLIPR/RBM operating system and the many real-time FORTRAN subroutines written by the CLIPR staff. The RBM monitor divides memory into concurrent foreground and background areas for the running of experiments and for program development and data analysis, respectively. This gives us a very responsive system for accurate reaction time measurement. Foreground can be further divided to run several experiments concurrently, with the possibility of running six experiments in foreground at the same time. Using multiple copies of the same program is much easier than writing a multiterminal executive, and it allows more flexibility in the mix of experiments being run at a given time. Thus, we can run six replications of the same experiment or six distinct experiments, each requiring a somewhat different program.

The FORTRAN real-time subroutines available make it possible to do many things that are usually considered impossible in FORTRAN. We have character manipulation routines, the ability to send and receive from digital $\mathrm{I} / \mathrm{O}$ devices, and the ability to measure reaction times to the nearest millisecond, taking actions based on the presence or absence of a response within a given time frame. We can dynamically allocate files and read from or write to disk files and terminals without the huge overhead FORTRAN normally imposes. Without these extensions to the language, we would be forced to do much of our programming at the assembly language level.

With experience, we have become aware of the elements common to our experiments. All studies involve running subjects in one of a number of conditions, describing the apparatus to the subject, describing the problem and its rules, recording moves that require multiple buttonpresses, and deciding what to do following the completion of each problem. From our early programming efforts, we have extracted the common elements, separated them from elements particular to a given problem, and developed a common skeleton for problem solving research. Now only minor changes need to be made to allow us almost infinite variations in the paradigm. The common skeleton consists of a series of modules called INITIATE, INSTRUCTION, PUZZLE, and ROUTING. The flow of control through the program is shown in Figure 1.

The INITIATE module does typical variable and data file initiation and then requests (from the experimenter) subject-identification information and experimental condition number. To save time and to eliminate a source of errors, the information describing the problems to be solved in a given condition is stored on a disk file constructed prior to running the experiment. Each condition in a given experiment is described by a record on this file containing various information. Typically, this includes such items as the specific problem to be presented, how the buttons should be labeled, where the instruction screens are stored, and how many problems are to be presented or what criterion is to be used for completion of the experiment.

Within each of the three general problem types, there have been a number of variations. For example, a number of Hobbits-Orcs problems have been used varying the number of pairs of characters to cross the river and the size of the boat. We also have used Tower of Hanoi problems in which the starting and goal pegs are varied, as well as the number of disks used. Within each problem class, the information needed to describe the particular problem is contained on the parameter file.

With the information in the parameter file read by the control program, we are permitted a great deal of flexibility. For example, we are able to give different groups of subjects isomorphic versions of a problem (i.e., versions with the same solution path, but with different descriptions) within a single program by simply changing the information about the instructions and the make-up of the problem display. Other variables enable us to have a single program for an entire class of problems; for example, for any Tower of Hanoi

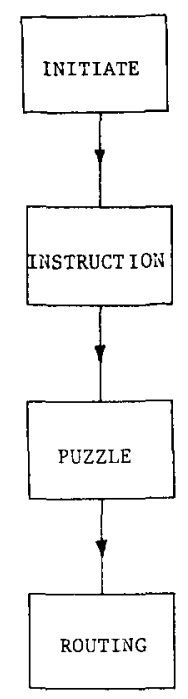

Figure 1. Flow diagram of experimental program. 
problem to be posed, the program need only be given three variables that determine the number of disks in the problem, and the start and goal states.

The INSTRUCTION module displays instructions to the subject. The instructions are a series of display screens that describe the nature of the problem and give directions on how to proceed. The subject can read the instructions at his own pace, paging either forward or backward through the instructions by using a button box in front of the CRT. All of our experiments use the CRT displays and accept input from a digital $\mathrm{I} / \mathrm{O}$ button box. As each instruction screen is read, the amount of time spent reading the screen is recorded. Between each problem solved, and between repeated solutions of the same problem, additional instructions may be given.

In early versions of the programs, we stored information about the location of the instruction screen file, and the instructions to be presented in each condition, in the program itself. This required us to alter the program for each slight manipulation made in the instruction sequence or content. In order to eliminate this problem, we now store not only the instruction file name, but also the number of instruction screens and their order on the parameter file described above. Changes in instructions are now accomplished without requiring the more cumbersome task of altering the program.

The PUZZLE modules present the individual problems while recording each move entered through the button box and its latency. Each move requires the subject to press a number of buttons. In the rivercrossing problems, one button is pressed for each character that the subject wishes to place in the boat (a variable number of buttonpresses are involved); in the water-jug and the Tower of Hanoi tasks, the subject presses two buttons to indicate movement from and to locations. The subject always has the option of cancelling a move after pressing the buttons before the move is carried out. The latencies between all buttonpresses are recorded by the program. Once a subject decides to enter a given move, it is evaluated for legality (all problems have the possibility that the subject might try to do something that is prohibited by the rules of the puzzle). The display is then altered to reflect the new problem state if the move is legal, or an error message is displayed if an illegal move has been attempted. Each puzzle program controls all variants of a class of tasks and allows for isomorphic problem versions. We currently have modules to control presentation of the missionaries-cannibals problems (Hobbits-Orcs), water-jug problems, the Hobbits-Orcs and Gold problem (a combined problem), and Tower of Hanoi problems.

The ROUTING module determines which problem, if any, the subject is to solve next, initializes the necessary variables, and passes control to the correct module. We currently allow for two types of problem solution sequences. A subject either solves the problem a fixed number of times or solves to a criterion of a given number of "perfect" (i.e., minimum-path) solutions. If the subject fails to solve a problem, a complicated sequence of events takes place, and this module oversees it also.

External to the run-time program, there is a data retrieval program that reformats a subject's data, stores them on tape, and produces a well formatted, annotated hard copy and summary. The real-time program outputs data in a format that is nearly identical for all problems, so that a new puzzle requires only minor modifications to the retrieval program.

The basic program design enables us to run experiments using almost any puzzle. We can introduce instructional manipulations trivially, and problem variants and isomorphs are also simple to implement. It is a more ambitious task to write a new puzzle control module, but much less work than programming an entire experiment from scratch.

The modules outlined above have been developed over the past 4 years and are still undergoing change. We started with special-purpose programs for each experiment; when we realized that our programs were proliferating, we extracted a common core from them as our initial skeleton. We have several caveats to offer other researchers. First, it is very important to make the program modular. Not only does this make the program easier to change, but when memory is at a premium, one can incorporate only those modules needed to run a particular experiment. Second, the program should be much more general than is expected to be necessary. In experimental work, it is common to find that each set of results suggests new experiments. Our ability to design the new experiments greatly exceeds the speed with which we write programs. One should never make a parameter constant if there is the slightest chance he or she may wish to someday vary it. If a routine is simple to incorporate, but there is no current need for it, it should be included anyway. Having the added flexibility may someday stimulate design considerations. Third, researchers should not underestimate the ingenuity of the bored subject. Consider every possible outcome of a test and include a procedure to handle its occurrence. There is no such thing as an outcome that will "never happen."

Our general problem solving program has been well worth the time spent on its development. It has decreased the time between the inception of an idea for an experiment and its execution from over 3 months to a few hours in many cases, and to 1 to 2 weeks in the worst case.

\section{REFERENCES}

Spear, T. L., Overgard, D., \& Christian, T. W. The ClipR display terminal experiment system. Behavior Research Methods \& Instrumentation, 1975, 7, 107-112.

STAdler, S. J. On the varieties of computer experience. Behavior Research Methodis \& Instrumentation. 1969, 1, 267-269. 